\title{
Radiotherapy for subfoveal neovascularisation associated with pathological myopia: a pilot study
}

\author{
Hiroshi Kobayashi, Kaori Kobayashi
}

\begin{abstract}
Backgroundlaim-Limited treatments are available for this disease process. A pilot study was performed to determine the toxicity and efficacy of external beam radiotherapy for subfoveal neovascular membranes and subretinal haemorrhage associated with pathological myopia.

Methods-A randomised, prospective study was carried out on 39 patients with subfoveal neovascularisation associated with high myopia. 20 patients underwent radiotherapy and the remaining 19 were observed as a randomised comparison group. All patients were followed up for at least 24 months. Subfoveal choroidal neovascular membranes (CNVMs) were treated with a single lateral $6 \mathrm{MV}$ photon beam to a dose of $\mathbf{1 0} \mathrm{Gy}$ in five fractions over 5-7 days. Post-treatment measurements included corrected visual acuity, area of CNVM, and occurrence of radiotherapy related complications, and adverse reactions. To assess changes of area of CNVM, the initial (pretreatment) size of the CNVM was set to $100 \%$, and all post-treatment measurements were normalised relative to the initial size.
\end{abstract}

Results-No significant acute morbidity was noted. There was no significant difference in age, sex, refractive error, visual acuity, and area of CNVM at baseline between the treatment group and control group. The mean change of the size of the CNVM for 2 years was $155 \%$ (SD $156 \%$ ) in the treatment group and $249 \%(124 \%)$ in the control group. The increase in the size of CNVM in the treatment group was significantly smaller than that in the control group $(p=0.0452)$. In the treated eyes, the visual acuity before and 1 and 2 years after radiotherapy were $0.111(22.2 / 200), 0.091(18.2 / 200)$, and 0.086 $(19.2 / 200)$, respectively. In the control eyes, visual acuity before and 1 and 2 years after the start of the follow up were 0.141 (34.2/200), $0.089(17.8 / 200)$, and $0.063(12.6 /$ 200). The patients in the treatment group showed no significant change for 2 years, and those in the control group showed a significant decrease in the visual acuity ( $p$ $=0.0033)$. The changes of $\log$ MAR of visual acuity for 2 years after the start of the follow up were $+0.019(0.443)$ in the treatment group and $+0.347(0.374)$ in the control group. There was a statistically significant difference between them ( $p=$ $0.0173)$. Multiple regression analysis on the treatment group showed that the most significant predictive variable for the visual acuity 2 years after the treatment was the combination of pretreatment visual acuity and refractive error.

Conclusions-Radiotherapy appeared to have a favourable treatment effect in eyes with subfoveal neovascular membranes and haemorrhage associated with pathological myopia. Further investigation is needed to evaluate the efficacy of radiotherapy for subfoveal neovascularisation associated with pathological myopia.

(Brf Ophthalmol 2000;84:761-766)

Patients with pathological myopia frequently develop slowly progressive loss of central acuity associated with myopic degenerative changes in the fifth or sixth decade. ${ }^{1}$ Fucks described a raised, circular, pigmented lesion that develops in the macula of middle aged patients (Foerster-Fucks spot). ${ }^{2}{ }^{3}$ This lesion consists of a localised ingrowth of fibrovascular tissue from the choroid and proliferation of pigment epithelial cells. In addition, bleeding from the subretinal neovascular membranes causes blurring or loss of central vision. No efficacious treatment for the subfoveal neovascularisation and its associated subretinal haemorrhage has been available. ${ }^{4-7}$

Several investigators demonstrated the efficacy and safety of low dose irradiation (10-20 Gy) for subfoveal choroidal neovascular membranes of age related macular degeneration. ${ }^{8-20}$ This study, for the first time, assesses low dose radiotherapy for subfoveal neovascular membrane and subretinal haemorrhage associated with pathologic myopia.

Subjects and methods

PATIENTS

Patients with high myopia were eligible for study when they had subfoveal neovascularisation. Indications for radiotherapy were (1) subfoveal choroidal neovascular membranes (CNVMs), (2) newly formed or exacerbated CNVMs, (3) visual acuity of $0.4(80 / 200)$ or less, (4) age of 60 and over, and (5) high myopia. High myopia was defined as the presence of myopic refraction of $-8 \mathrm{D}$ or over and axial length of $26 \mathrm{~mm}$ or longer in the presence of typical fundal changes of high myopia. Thirty nine patients with subfoveal neovascularisation on fluorescein angiography were identified for inclusion into the study. The angiograms of all these patients showed early leakage of dye seen as hyperfluorescence which involved the foveal avascular zone. Any patient with pre-existing ocular disease (glaucoma, chronic inflammatory, or neoplastic disorders) was excluded as 
were those with systemic disorders (diabetes, uncontrolled hypertension) or a known life threatening disease at enrolment into the study. Patients with signs consistent with age related macular degeneration, including soft drusen and retinal pigment epithelium (RPE) abnormalities, were excluded.

The study protocol and consent forms were approved by the human subjects committee of Amagasaki Hospital. Patients were informed of the purpose of our study, and provided their signed consent to participate. Thirty nine patients were prospectively randomised to radiotherapy or no treatment, with only one eye of a patient to be randomised. For cases in which we believed that both eyes of a patient were eligible, we and the patient made a subjective judgment as to which eye would be enrolled in the study. Within 24 hours after enrolment, the patients were randomised using computer generated numbers; 0 is to receive low dose radiotherapy and 1 is to receive no treatment. The patients' randomisation state was masked to the treating doctor (HK). Twenty patients underwent radiotherapy, and the remaining 19 patients who did not undergo radiotherapy were followed up as a randomised group. Within a week after random assignments treatment was to begin.

All recruited patients underwent a complete ophthalmological examination, including measurement of the corrected visual acuity, near vision, slit lamp biomicroscopy, and indirect ophthalmoscopy. Fully corrected distance visual acuity was measured on the Early Treatment Diabetic Retinopathy Study (ETDRS) chart. The logarithm of the minimum angle of resolution (logMAR) was calculated and used for all statistical analysis. An immediate pretreatment fluorescein angiogram was obtained within 1 week of commencing radiotherapy. Radiotherapy was carried out as described as below. After radiotherapy the patients were reviewed at 2 weeks, 1, 2, 3, 6, 9, $12,18,24$ months, and then yearly. Safety was evaluated by determining the incidence of radiotherapy related complications and adverse reactions for both treatment and control groups. The patients were regularly questioned and examined for side effects. The control group received the same follow up as the treatment group. Visual function was assessed at every visit and angiography scheduled for the $3,6,12,18$, and 24 month visits. Assessment of outcomes including visual acuity, angiographic interpretation, assessment of complications, and adverse events was performed in a masked fashion. To detect adverse events, all patients were examined by the same examiner (HK) to avoid interobserver variation. For assessment of cataract, nuclear sclerosis was assessed as described by Emery et al..$^{21}$ Anterior and posterior subcapsular cataract and cortical opacity were scored according to Heckenlively. ${ }^{22}$

RADIOTHERAPY

Patients were immobilised with a thermoplastic mask with the head in normo-extension. The isocentre was determined in the centre of the radiotherapy eye, $15 \mathrm{~mm}$ behind the cornea. Irradiation was administered with 6 MV photons with a gantry position of 90 degrees. A high definition computed tomography (CT) scan of the orbit was obtained. Cursor measurements were made from surface markers placed on the Perspex shell at the temporal region and the position of the posterior pole of the eye plotted from these measurements. Computer generated isodose curves for a single $6 \mathrm{MV}$ photon beam, given to $90 \%$ of the maximum dose, were superimposed onto the CT scan images. The 90\% isodose encompassed the macula of the affected eye. All patients received a dose of $10 \mathrm{~Gy}$ prescribed to the $90 \%$ isodose, delivered as five fractions of 2 Gy over 5-7 days. All eyes were irradiated through a single lateral port.

\section{DETERMINATION OF AREA OF CNVM}

A CNVM associated with pathological myopia showed the area of hyperfluorescence with well boundaries, allowing for accurate determination of the location and size of the lesion. Measurements of the CNVM included only CNVM, but not contiguous blood, fibrosis, or atrophy. In pathological myopia, subretinal blood was thin and did not obscure the boundaries of the CNVM, whereas subretinal blood was thick and obscured the CNVM in age related macular degeneration. Subretinal blood was considered to be present if blood was under the retina in a location immediately contiguous with the area of CNVM or within a serous sanguineous detachment of the retina contiguous with the area of CNVM. Fundus angiograms were obtained using a high resolution digital fundus imaging system based on a UVi fundus camera (Canon, Tokyo, Japan). In each angiogram, one picture was selected that gave the extent of the CNVM and the optic disc. The image was analysed with UTHSCSA Image Tool 32 bit image analysis program (developed at the University of Texas Health Science Center at San Antonio, TX, USA). After applying sharpening and contrast enhancing image filters, the outline of the membrane was drawn on the image manually and the membrane surface was calculated. The outline of the optic disc was simultaneously drawn and saved. To calculate for magnification errors, the disc/CNVM ratio was calculated for each image. The initial (pretreatment) size of the CNVM was set to $100 \%$, and all post-treatment measurements were normalised relative to the initial size. When repeated measurement done on the same picture, statistical analysis of the reproducibility of this measuring method showed a high correlation coefficient of 0.995 . A change of less than $20 \%$ was considered as unchanged.

\section{STATISTICAL ANALYSIS}

Values are presented as the mean (SD) and range. Unless otherwise specified, data were analysed by paired, unilateral $t$ tests. A level of $\mathrm{p}<0.05$ was accepted as statistically significant.

Stepwise regression analysis on both the treatment group and control group was used to determine which factors the visual acuity, age, refractive error, and area of CNVM at baseline 
would predict the visual acuity and area of CNVM at 2 years after the start of the follow up. Regression analysis was performed to determine the precise relation between variables. For regression analysis, a level of $\mathrm{p}<0.01$ was accepted as statistically significant.

\section{Results}

AT BASELINE

Table 1 shows a comparison of age, sex, and refractive error in the treatment group and control group at baseline, and there was no significant difference between two groups. Baseline visual acuity was slightly poorer in the treatment group compared with untreated, but this difference was not statistically significant. Mean area of pretreatment CNVM was 1.472 (1.055) $\mathrm{mm}^{2}$ in the treatment group and 1.549 (1.065) $\mathrm{mm}^{2}$ in the control group. No difference was found between two groups. Fourteen patients $(70 \%)$ in the treatment group and 16 patients $(84 \%)$ in the control group exhibited lacquer cracks at the site of the CNVM.

POSSIBLE ADVERSE REACTIONS

Throughout the study, patients were monitored for any possible adverse side effects which could be attributed to radiotherapy. In the treatment group, one patient suffered transient conjunctival irritation with resolution with 2 weeks and thereafter has remained asymptomatic. No significant progression of cortical and posterior subcapsular lens opacities was observed. Radiation induced retinal vasculopathy (microvascular abnormalities, leakage, and cotton wool spots) or optic neuropathy (disc pallor) were not observed clinically. Angiograms were scrutinised for evidence of retinal microvascular abnormalities and none was found. In the control group, no complication or adverse reaction was found.

AT FOLLOW UP

Mean changes of area of the CNVM for 1 and 2 years after the start of the follow up were $145 \%(95 \%)$ and $155 \%(156 \%)$ in the treatment group and $208 \%(98 \%)$ and $249 \%$ $(124 \%)$ in the control group, respectively (Table 2). The increase in the area of CNVM in the treatment group for 2 years was significantly smaller than that in the control group ( $p=0.0452)$. Of 19 patients in the control group, one patient showed regression of the CNVM and four patients showed stable fluorescein angiographic appearance at 2 years after the start of the follow up (Table 2). Of 20 patients in the treatment group, seven patients showed significant regression and six patients showed stable fluorescein angiographic appearance at 2 years after the radiotherapy. All patients with subretinal haemorrhage showed complete resolution in the treatment group (Table 2).

In the treated eyes, mean visual acuity before and 1 and 2 years after radiotherapy were $0.111(22.2 / 200), 0.091(18.2 / 200)$, and 0.086 $(19.2 / 200)$, respectively (Table 1$)$. No significant difference was found between each two of three examinations. In the control eyes, mean visual acuity before and 1 and 2 years after the

Table 1 Demographics of patient groups

\begin{tabular}{|c|c|c|c|}
\hline & Treatment group & Control group & $p$ Value \\
\hline No of patients & 20 & 19 & \\
\hline \multicolumn{4}{|l|}{ Baseline } \\
\hline Men & $6(30 \%)$ & $5(26 \%)$ & 0.7982 \\
\hline Women & $14(70 \%)$ & $14(74 \%)$ & \\
\hline Right eye & $11(55 \%)$ & $11(58 \%)$ & 0.8554 \\
\hline Left eye & $9(45 \%)$ & $8(42 \%)$ & \\
\hline \multicolumn{4}{|l|}{ Age (years) } \\
\hline $60-69$ & $8(40 \%)$ & $9(47 \%)$ & \\
\hline $70-79$ & $10(50 \%)$ & $9(47 \%)$ & \\
\hline $80-89$ & $2(10 \%)$ & $1(5 \%)$ & \\
\hline Mean (SD) & $72.90(6.52)$ & $72.37(7.16)$ & 0.8102 \\
\hline (range) & $(60-89)$ & $(60-89)$ & \\
\hline \multicolumn{4}{|l|}{ Refractive error (D) } \\
\hline-8 to -10 & 9 eyes $(45 \%)$ & 10 eyes $(53 \%)$ & \\
\hline-10.1 to -15 & 8 eyes $(40 \%)$ & 7 eyes $(37 \%)$ & \\
\hline-15.1 to -20 & 2 eyes $(10 \%)$ & 1 eye $(5 \%)$ & \\
\hline$<-21$ & 1 eye $(5 \%)$ & 1 eye $(5 \%)$ & \\
\hline Mean (SD) & $-11.73(3.89)$ & $-11.33(4.74)$ & \\
\hline (range) & $(-22.0$ to -8.0$)$ & $(-28.0$ to -8.0$)$ & 0.7744 \\
\hline \multicolumn{4}{|l|}{ Pretreatment area of CNVM } \\
\hline $0-0.250 \mathrm{~mm}^{2}$ & 2 eyes $(10 \%)$ & 2 eyes $(11 \%)$ & \\
\hline $0.251-0.500 \mathrm{~mm}^{2}$ & 4 eyes $(20 \%)$ & 2 eyes $(11 \%)$ & \\
\hline $0.501-1.000 \mathrm{~mm}^{2}$ & 3 eyes $(15 \%)$ & 4 eyes $(21 \%)$ & \\
\hline $1.001-2.000 \mathrm{~mm}^{2}$ & 5 eyes $(25 \%)$ & 4 eyes $(21 \%)$ & \\
\hline $2.001-3.000 \mathrm{~mm}^{2}$ & 3 eyes $(15 \%)$ & 3 eyes $(16 \%)$ & \\
\hline $3.001-4.000 \mathrm{~mm}^{2}$ & 3 eyes $(15 \%)$ & 4 eyes $(21 \%)$ & \\
\hline Mean (SD) & $1.472(1.055)$ & $1.549(1.065)$ & 0.8218 \\
\hline (range) & $(0.221$ to 3.554$)$ & $(0.105$ to 3.331$)$ & \\
\hline \multicolumn{4}{|l|}{ Subretinal haemorrhage } \\
\hline$(+)$ & $11(55 \%)$ & $9(47 \%)$ & 0.6336 \\
\hline$(-)$ & $9(45 \%)$ & $10(53 \%)$ & \\
\hline \multicolumn{4}{|l|}{ Visual acuity } \\
\hline $0.1-0.5(20 / 200-20 / 40)$ & 7 eyes $(35 \%)$ & 9 eyes $(47 \%)$ & \\
\hline $0.05-0.09(20 / 400-20 / 222)$ & 5 eyes $(25 \%)$ & 5 eyes $(26 \%)$ & \\
\hline $0.01-0.04(20 / 2000-20 / 500)$ & 8 eyes $(40 \%)$ & 5 eyes $(26 \%)$ & \\
\hline Mean & $0.111(22.2 / 200)$ & $0.141(34.2 / 200)$ & \\
\hline (range) & $(0.01$ to 0.4$)$ & $(0.02$ to 0.4$)$ & \\
\hline Mean (SD) (logMAR) & $0.954(0.595)$ & $0.851(0.403)$ & 0.5329 \\
\hline
\end{tabular}

LogMAR of visual acuity $=$ logarithm of the minimal angle resolution of visual acuity. CNVM = choroidal neovascular membrane. Refractive error was the spherical equivalent. 
Table 2 Changes of visual acuity and area of CNVM after follow up

\begin{tabular}{|c|c|c|c|}
\hline & Treatment group & Control group & p Value \\
\hline No of patients & 20 & 19 & \\
\hline Duration of follow up (months) & $27.2(4.6)(24$ to 36$)$ & $28.9(5.2)(24$ to 38$)$ & 0.2859 \\
\hline \multicolumn{4}{|l|}{ Area of CNVM $\left(\mathrm{mm}^{2}\right)$} \\
\hline Baseline & $1.472(1.055)(0.221$ to 3.554$)$ & $1.549(1.065)(0.105$ to 3.331$)$ & 0.8218 \\
\hline 3 months & $1.584(1.097)(0.245$ to 3.983$)$ & $1.956(1.332)(0.235$ to 4.244$)$ & 0.3488 \\
\hline 6 months & $1.601(1.102)(0.287$ to 4.488$)$ & $2.133(1.367)(0.332$ to 5.301$)$ & 0.1880 \\
\hline 12 months & $1.619(1.122)(0.301$ to 4.807$)$ & $2.201(1.439)(0.406$ to 5.396$)$ & 0.1661 \\
\hline 24 months & $1.317(1.435)(0.422$ to 5.533$)$ & $2.717(1.974)(0.496$ to 7.395$)$ & 0.0153 \\
\hline \multicolumn{4}{|l|}{ Change of area of CNVM } \\
\hline 3 months & $117.61(78.36) \%(78$ to $188 \%)$ & $136.28(80.28) \%(83$ to $287 \%)$ & 0.4670 \\
\hline 6 months & $138.76(84.22) \%(71$ to $245 \%)$ & $156.35(87.72) \%(83$ to $332 \%)$ & 0.5271 \\
\hline 12 months & $144.67(94.59) \%(54$ to $351 \%)$ & $208.37(98.32) \%(78$ to $411 \%)$ & 0.2823 \\
\hline 24 months & $154.87(156.49) \%(35$ to $632 \%)$ & $248.84(123.83) \%(77$ to $472 \%)$ & 0.0452 \\
\hline \multicolumn{4}{|c|}{ Eyes with change of area of CNVM 24 months after the start of the follow up } \\
\hline Decreased $(\geqslant 20 \%)$ & 7 eyes $(35 \%)$ & 1 eyes $(5 \%)$ & \\
\hline Unchanged & 6 eyes $(30 \%)$ & 4 eyes $(21 \%)$ & \\
\hline Increased $(\geqslant 20 \%)$ & 7 eyes $(35 \%)$ & 14 eyes $(74 \%)$ & \\
\hline \multicolumn{4}{|c|}{ Area of CNVM 24 months after the start of the follow up } \\
\hline $0-0.250 \mathrm{~mm}^{2}$ & 1 eye $(5 \%)$ & 0 eye $(0 \%)$ & \\
\hline $0.251-0.500 \mathrm{~mm}^{2}$ & 3 eyes $(15 \%)$ & 1 eye $(5 \%)$ & \\
\hline $0.501-1.000 \mathrm{~mm}^{2}$ & 4 eyes $(20 \%)$ & 4 eyes $(21 \%)$ & \\
\hline $1.001-2.000 \mathrm{~mm}^{2}$ & 9 eyes $(45 \%)$ & 4 eyes $(21 \%)$ & \\
\hline $2.001-3.000 \mathrm{~mm}^{2}$ & 2 eyes $(10 \%)$ & 2 eyes $(11 \%)$ & \\
\hline $3.001-4.000 \mathrm{~mm}^{2}$ & 0 eye $(0 \%)$ & 3 eyes $(16 \%)$ & \\
\hline $4.001-5.000 \mathrm{~mm}^{2}$ & 1 eye $(5 \%)$ & 2 eyes $(11 \%)$ & \\
\hline$>5.000 \mathrm{~mm}^{2}$ & 0 eye $(0 \%)$ & 1 eye $(5 \%)$ & \\
\hline \multicolumn{4}{|l|}{ Visual acuity } \\
\hline Baseline & $0.111(22.2 / 200)(0.01$ to 0.4$)$ & $0.141(34.2 / 200)(0.02$ to 0.4$)$ & \\
\hline LogMAR & $0.958(0.470)$ & $0.851(0.403)$ & \\
\hline 3 months & $0.120(24 / 200)(0.02$ to 0.4$)$ & $0.120(24 / 200)(0.03$ to 0.4$)$ & \\
\hline LogMAR & $0.922(0.362)$ & $0.921(0.394)$ & \\
\hline 6 months & $0.108(21.6 / 200)(0.02$ to 0.4$)$ & $0.106(21.2 / 200)(0.04$ to 0.4$)$ & \\
\hline LogMAR & $0.964(0.333)$ & $0.971(0.319)$ & \\
\hline 12 months & $0.091(18.2 / 200)(0.02$ to 0.6$)$ & $0.089(17.8 / 200)(0.01$ to 0.2$)$ & \\
\hline LogMAR & $1.006(0.338)$ & $1.049(0.278)$ & \\
\hline 24 months & $0.086(19.2 / 200)(0.02$ to 0.6$)$ & $0.063(12.6 / 200)(0.01$ to 0.2$)$ & \\
\hline LogMAR & $0.977(0.353)$ & $1.198(0.264)$ & \\
\hline \multicolumn{4}{|c|}{ Change of $\log$ MAR visual acuity after the start of the follow up } \\
\hline 3 months & $-0.035(0.262)(-0.602$ to +0.523$)$ & $+0.070(0.237)(-0.699$ to +0.477$)$ & 0.1982 \\
\hline 6 months & $+0.007(0.365)(-0.477$ to +0.903$)$ & $+0.120(0.253)(-0.699$ to +0.$)$ & 0.2708 \\
\hline 12 months & $+0.048(0.426)(-0.477$ to +1.125$)$ & $+0.198(0.290)(-0.301$ to +0.699$)$ & 0.2090 \\
\hline 24 months & $+0.019(0.443)(-0.477$ to 1.301$)$ & $+0.347(0.374)(-0.477$ to +0.602$)$ & 0.0173 \\
\hline \multicolumn{4}{|c|}{ Eyes with change of visual acuity during 24 months } \\
\hline Improved ( $\geqslant 2$ lines) & 9 eyes $(45 \%)$ & 3 eyes $(16 \%)$ & \\
\hline Unchanged & 4 eyes $(20 \%)$ & 3 eyes $(16 \%)$ & \\
\hline Deteriorated ( $\geqslant 2$ lines) & 7 eyes $(35 \%)$ & 13 eyes $(68 \%)$ & \\
\hline \multicolumn{4}{|c|}{ Visual acuity 24 months after the start of the follow up } \\
\hline$\geqslant 0.6(120 / 200)$ & 1 eye $(5 \%)$ & 0 eye $(0 \%)$ & \\
\hline 0.1 to $0.5(20 / 200$ to $100 / 200)$ & 12 eyes $(60 \%)$ & 5 eyes $(26 \%)$ & \\
\hline 0.06 to $0.09(12 / 200$ to $18 / 200)$ & 3 eyes $(15 \%)$ & 4 eyes $(21 \%)$ & \\
\hline 0.01 to $0.05(2 / 200$ to $10 / 200)$ & 4 eyes $(20 \%)$ & 10 eyes $(53 \%)$ & \\
\hline
\end{tabular}

LogMAR of visual acuity $=$ logarithm of the minimal angle resolution of visual acuity. CNVM = choroidal neovascular membrane.

start of the follow up were $0.141(34.2 / 200)$, $0.089(17.8 / 200)$, and $0.063(12.6 / 200)$, respectively. The visual acuity significantly de-

Table 3 Regression analysis and correlation coefficients for visual acuity and area of CNVM 2 years after the start of the follow up, and their changes

\begin{tabular}{|c|c|c|c|c|}
\hline & \multicolumn{2}{|c|}{$\begin{array}{l}\text { Treatment group } \\
(N=20)\end{array}$} & \multicolumn{2}{|c|}{ Control group $(N=19)$} \\
\hline & $r$ & p Value & $r$ & p Value \\
\hline \multicolumn{5}{|c|}{ LogMAR of visual acuity 2 years after the start of the follow up } \\
\hline LogMAR of pretreatment visual acuity & 0.598 & 0.0323 & 0.532 & 0.0247 \\
\hline Age & 0.360 & 0.1872 & 0.030 & 0.9018 \\
\hline Refractive error & 0.432 & 0.1076 & 0.283 & 0.2412 \\
\hline Area of CNVM & 0.101 & 0.7195 & 0.297 & 0.2170 \\
\hline \multicolumn{5}{|c|}{ Change of logMAR of visual acuity for 2 years } \\
\hline LogMAR of pretreatment visual acuity & 0.713 & 0.0028 & 0.796 & 0.0001 \\
\hline 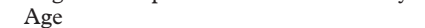 & 0.408 & 0.1308 & 0.115 & 0.6381 \\
\hline Refractive error & 0.354 & 0.1949 & 0.054 & 0.8262 \\
\hline Area of CNVM & 0.532 & 0.0411 & 0.371 & 0.1176 \\
\hline \multicolumn{5}{|c|}{ Area of CNVM 2 years after after the start of the follow up } \\
\hline LogMAR of pretreatment visual acuity & 0.116 & 0.6811 & 0.013 & 0.9565 \\
\hline Age & 0.012 & 0.9661 & 0.147 & 0.5437 \\
\hline Refractive error & 0.352 & 0.1984 & 0.394 & 0.0947 \\
\hline Area of CNVM & 0.630 & 0.0180 & 0.718 & 0.0005 \\
\hline \multicolumn{5}{|l|}{ Change of area of CNVM for 2 years } \\
\hline LogMAR of pretreatment visual acuity & 0.256 & 0.3569 & 0.506 & 0.0272 \\
\hline Age & 0.270 & 0.3313 & 0.268 & 0.2627 \\
\hline Refractive error & 0.366 & 0.1795 & 0.113 & 0.6443 \\
\hline Area of CNVM & 0.540 & 0.0378 & 0.538 & 0.0175 \\
\hline
\end{tabular}

LogMAR of visual acuity $=$ logarithm of the minimal angle resolution of visual acuity. CNVM $=$ choroidal neovascular membrane. creased for 2 years $(p=0.0033)$. The changes of $\log$ MAR of visual acuity for 2 years after the start of the follow up were $+0.019(0.443)$ in the treatment group and $+0.347(0.374)$ in the control group. There was a statistically significant difference between them $(p=0.0173)$.

FACTORS INFLUENCING CHANGES OF CNVM SIZE AND VISUAL ACUITY AFTER RADIOTHERAPY In the treatment group, there were a significant correlation of changes in visual acuity and pretreatment visual acuity $(r=0.713 ; \mathrm{p}=0.0028)$, as shown in Table 3. Multiple regression analysis showed that the most significant predictive variable of visual acuity at 2 years after the treatment was the combination of pretreatment visual acuity and refractive error and then pretreatment visual acuity only (Table 4 ). The strongest factor for the prediction of area of CNVM at 2 years after the treatment was pretreatment of the CNVM area.

\section{Discussion}

We demonstrated the safety and efficacy of radiotherapy for a subfoveal neovascular membrane associated with pathological myopia. 
Table 4 Multiple regression analysis of predictors of visual acuity and area of CNVM 2 years after the start of the follow up, and their changes

\begin{tabular}{|c|c|c|c|}
\hline Variable entered & $r$ & F test & Correlation equation \\
\hline \multicolumn{4}{|l|}{ Treatment group $(N=20)$} \\
\hline \multirow[t]{2}{*}{ Visual acuity at 2 years after radiotherapy } & 0.598 & 4.295 & $\begin{array}{l}\text { [LogMAR of post-treatment visual acuity }]=+0.649+0.361 \times[\log M A R \text { of } \\
\text { pretreatment visual acuity }]\end{array}$ \\
\hline & 0.661 & 4.660 & $\begin{array}{l}\text { [LogMAR of post-treatment visual acuity }]=+0.649+0.362 \times[\log M A R \text { of } \\
\text { pretreatment visual acuity }]-0.043 \times[\text { refractive error }]\end{array}$ \\
\hline \multirow[t]{2}{*}{ Change of visual acuity } & 0.713 & $\begin{array}{c}13.47 \\
5\end{array}$ & $\begin{array}{l}\text { [Change of } \log \text { MAR of post-treatment visual acuity }]=+0.649-0.369 \times[\log \text { MAR of } \\
\text { pretreatment visual acuity] }\end{array}$ \\
\hline & 0.795 & $\begin{array}{c}10.31 \\
8\end{array}$ & $\begin{array}{l}\text { [Change of logMAR of post-treatment visual acuity }]=+1.178-0.638 \times[\log \text { MAR of } \\
\text { pretreatment visual acuity }]-0.043 \times[\text { refractive error }]\end{array}$ \\
\hline Area of $\mathrm{CNVM}$ at 2 years after radiotherapy & 0.630 & 8.561 & {$[$ Post-treatment area of CNVM] $=0.648+0.479 \times[$ pretreatment area of CNVM] } \\
\hline Change of area of CNVM & 0.540 & 5.349 & [Change of area of CNVM] $=259.732-69.54 \times[$ pretreatment area of CNVM] \\
\hline \multicolumn{4}{|l|}{ Control group $(N=19)$} \\
\hline Visual acuity at 2 years after the start of the follow up & - & - & - \\
\hline Change of visual acuity & 0.796 & 29.37 & $\begin{array}{l}\text { [Change of } \log M A R \text { of post-treatment visual acuity }]=+0.994-0.748 \times[\log M A R \text { of } \\
\text { pretreatment visual acuity }]\end{array}$ \\
\hline \multirow[t]{2}{*}{ Area of CNVM at 2 years after the start of the follow up } & 0.839 & $\begin{array}{l}40.40 \\
3\end{array}$ & $\begin{array}{l}\text { [Post-treatment area of CNVM] }=1.171+1.403 \times[\text { pretreatment area of CNVM] }- \\
1.344 \times[\text { LogMAR of pretreatment visual acuity }]\end{array}$ \\
\hline & 0.898 & $\begin{array}{l}33.15 \\
8\end{array}$ & {$[$ Post-treatment area of CNVM] $=0.445+1.133 \times[$ pretreatment area of CNVM] } \\
\hline Change of area of CNVM & 0.538 & 6.924 & {$[$ Change of area of CNVM] $=315.7-62.6 \times[$ pretreatment area of CNVM] } \\
\hline
\end{tabular}

LogMAR of visual acuity $=$ logarithm of the minimal angle resolution of visual acuity. CNVM = choroidal neovascular membrane.

The increases in the area of CNVM in the treatment group for 2 years were significantly smaller than those in the control group. The patients in the treatment group showed no significant change in the visual acuity for 2 years, and those in the control group showed a significant decrease.

Tokoro showed that $50 \%$ of eyes with CNVM had visual acuity less than 0.1 and $75 \%$ had visual acuity less than 0.3 , although the percentage of CNVM is low in eyes with visual acuity less than $0.01 .^{3}$ Because the distribution of visual acuity of eyes in this study was similar to those reported by Tokoro, visual acuity at baseline was relatively low.

There is a potential risk of development of malignancy induced by radiotherapy in younger patients in spite of the low dose. We enrolled patients at the age of 60 or over, because these patients have a minimal risk for carcinogenesis.

The guidelines for laser photocoagulation treatment for subretinal neovascularisation caused by other diseases may not be applicable to treatment of this complication in patients with myopic degeneration. ${ }^{4}$ The extreme thinness of the choroid and Bruch's membrane in eyes with myopic degeneration makes them particularly vulnerable to mechanical effects, including those induced by photocoagulation. This together with our success with radiotherapy suggests that a CNVM associated with pathological myopia may be treated with radiotherapy.

Although a CNVM associated with age related macular degeneration needs a dose of 15-20 Gy of irradiation, ${ }^{8-20}$ a dose of $10 \mathrm{~Gy}$ may be enough dose to treat the subretinal neovascular membrane with pathological myopia because of the relatively limited size of the membrane. ${ }^{1-3}$ In this study, we used a dose of $10 \mathrm{~Gy}$ in fraction of $2 \mathrm{~Gy}$. The risk of optic neuropathy and radiation induced retinopathy is significantly increased with larger fraction sizes. If recurrence is observed, radiotherapy can be performed again. One patient in the treatment group showed regrowth of CNVM with subretinal haemorrhage 26 months after radiotherapy. The patient underwent an additional irradiation of $10 \mathrm{~Gy}$, which induced regression of the CNVM and resolution of the haemorrhage. However, it is not still clear whether a dose of $10 \mathrm{~Gy}$ is enough for the treatment of a CNVM. A further investigation regarding dose, including an escalation study, is required to determine the dose effective for the CNVMs.

To evaluate the efficacy of radiotherapy for subfoveal CNVM associated pathological myopia is more difficult than for CNVM associated with age related macular degeneration. In a natural course, no patient with age related macular degeneration show regression of CNVM. ${ }^{1}$ Of 19 patients in this study, one patient with high myopia showed spontaneous regression of CNVM and eight showed resolution of subretinal haemorrhage. There is little literature regarding to the long term observation of the subretinal CNVM associated pathological myopia. Before new formation or exacerbation of CNVMs, eyes with high myopia usually show myopic degenerations and loss of vision. In the course of the follow up, the progression of the degenerations may lead to further decrease of visual acuity and make it difficult to evaluate the effects of treatment. Therefore, more cases are needed to evaluate the efficacy of radiotherapy.

In this study, multiple regression analysis demonstrated that the most significant predictive factor for visual acuity 2 years after the treatment was the combination of pretreatment visual acuity and refractive error and then pretreatment visual acuity only. The strongest variable for the prediction of change of CNVM area was pretreatment CNVM area. The ability to predict the post-treatment visual results would be helpful in treating patients with subfoveal neovascularisation associated with pathological myopia.

In this study, we demonstrated the safety and efficacy of radiotherapy for subfoveal neovascularisation and subretinal haemorrhage associated with pathological myopia. Long term 
follow up is required to assess side effects, including cataract, radiation induced retinopathy, and optic neuropathy, as well as recurrence of subretinal neovascularisation.

1 Gass JDM. Stereoscopic atlas of macular diseases: diagnosis and treatment. 4th ed. St Louis: CV Mosby, 1997:162-9.

2 Fucks E. Der centrale schwarze fleck bei Myopie. Z Augenheilkd 1901;5:171-81.

3 Tokoro K. Atlas of posterior fundus changes in pathologic myopia. Tokyo: Springer-Verlag, 1998.

4 Jalkh AE, Weiter JJ, Trempe CL, et al. Choroidal neovascularization with degenerative myopia: role of laser photocoagulation. Ophthalmic Surg 1987;18:721-5.

5 Thomas MA, Grand MG, Williams DF, et al. Surgical management of subfoveal choroidal neovascularization. $\mathrm{Oph}-$ thalmology 1992;99:952-68.

6 Joseph DP, Thomas MA. Present indications for removal of choroidal neovascular membranes. Curr Opin Ophthalmol 1998;9:11-215.

7 Berger AS, Kaplan AG. Clinical experience with the surgical removal of sub-foveal neovascular membranes. Ophthalmolremoval of sub-foveal
ogy 1992;99:969-74.

8 Chakravarthy U, Houston RF, Archer DB. Treatment of age-related subfoveal neovascular membranes by age-related subfoveal neovascular membranes by
teletherapy: a pilot study. BrfOphthalmol 1993;77:265-73.

9 teletherapy: a pilot study. Br f Ophthalmol 1993;77:265-73. Hart PM, Chakraverthy U, MacKenzie G, et al. Therapy for
subfoveal choroidal neovascularization of age-related macular degeneration: results of follow up in a nonrandomised study. $\mathrm{Br} \mathcal{F}$ Ophthalmol 1996;80:1046-50.

10 Bergink GJ, Deutman AF, van den Broek JFCN, et al. Radiation therapy for subfoveal choroidal neovascular membranes in age-related macular degeneration. Graefes Arch Clin Exp Ophthalmol 1994;262:591-8.

11 Berson AM, Finger PT, Sherr DL, et al. Radiotherapy for age-related macular degeneration: preliminary results of a potentially new treatment. Int $\mathcal{F}$ Radiat Oncol Biol Phys 1996;36:861-5.
12 Finger PT, Berson A, Sherr D, et al. Radiation therapy for Finger PT, Berson A, Sherr D, et al. Radiation therapy for subretin 89 .

13 Freire J, Longton WA, Miyamoto CT, et al. External radiotherapy in macular degeneration: technical and preliminary subjective response. Int $\mathcal{F}$ Radiat Oncol Biol Phys 1996;36: $857-9$.

14 Valmaggia C, Bischoff P, Ries G. Niedrig dosierte Radiotherapie der subfoveolären Neovaskularisationen bei altersabhängiger Makuladegeneration. Resultate nach 6 Wochen und 6 Monate. Klin Monatsbl 1996;208:315-17.

15 Hollick EJ, Goble RR, Knowles PJ, et al. Radiotherapy treatment of age-related subfoveal neovascular membranes in patients with good vision. Eye 1996;10:609-16.

16 Yonemoto LT, Slater JD, Friedchsen EJ, et al. Phase I/II study of proton beam irradiation for the treatment of subfoveal choroidal neovascularization in age-related macular degeneration: treatment techniques and preliminary results. Int f Radiat Oncol Biol Phys 1996;36:867-91.

17 Brady LW, Freire JE, Longton WA, et al. Radiation therapy for macular degeneration: technical considerations and for macular degeneration: technical considerations and
preliminary results. Int $\mathcal{F}$ Radiat Oncol Biol Phys 1997;39: preliminary.

18 Sasai K, Murata R, Mandai M, et al. Radiation therapy for cular choroidal neovascularization liminary study. Int 7 Radiat Oncol Biol Phys 1997;39:173-8.

19 Stalmans P, Leys A, Van Limbergen E. External beam radiotherapy (20 Gy, 2 Gy fractions) fails to control the growth of choroidal neovascularization in age-related macular degeneration. Retina 1997;17:481-92.

20 Spaide RF, Guyer DR, McCormick B, et al. External beam radiation therapy for choroidal neovascularization. Ophthalmology 1998;105:24-30.

21 Emery JM, et al. Phacoemulsification and aspiration of cataract: surgical techniques, complications, and results. St Louis: CV Mosby, 1979:45-8.

22 Heckenlively JR. Frequency of posterior subcapsular cataract in the hereditary retinal degenerations. $\mathrm{Am} \mathcal{F} \mathrm{Oph}$ thalmol 1982;93:733-8. 